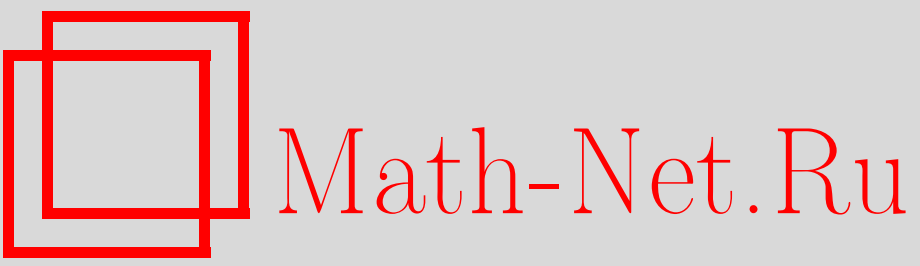

С. С. Герштейн, А. А. Логунов, М. А. Мествиришвили, О внутреннем решении типа Шварцшильда в полевой теории гравитации, ТМФ, 2006, том 147, номер 1, 155-160

DOI: https://doi.org/10.4213/tmf2030

Использование Общероссийского математического портала Math-Net.Ru подразумевает, что вы прочитали и согласны с пользовательским соглашением

http://www.mathnet.ru/rus/agreement

Параметры загрузки:

IP: 54.209.52.79

26 апреля 2023 г., 18:31:20 


\title{
ФИЗИКА
}

Том 147, № 1

апрель, 2006

(C) 2006 г.

С. С. Герштейн*, А. А. Логунов*, М. А. Мествиришвили*

\section{О ВНУТРЕННЕМ РЕШЕНИИ ТИПА ШВАРЦШИЛЬДА В ПОЛЕВОЙ ТЕОРИИ ГРАВИТАЦИИ}

\begin{abstract}
Показано, что внутреннее решение типа Шварцшильда в полевой теории гравитации не приводит к бесконечному давлению внутри тела, как это имеет место в общей теории относительности. Это происходит благодаря массе покоя гравитона из-за остановки процесса замедления хода времени.
\end{abstract}

Ключевые слова: гравитационная сила отталкивания, замедление хода времени, масса покоя гравитона.

Шварцшильд в статьях [1] нашел сферически-симметричные статические (внешнее и внутреннее) решения уравнений общей теории относительности (ОТО). Внешнее решение широко известно и имеет следующий вид:

$$
d s^{2}=c^{2}\left(1-\frac{W_{g}}{W}\right) d t^{2}-\left(1-\frac{W_{g}}{W}\right)^{-1} d W^{2}+W^{2}\left(d \theta^{2}+\sin ^{2} \theta d \phi^{2}\right),
$$

где $W_{g}=(2 G M) / c^{2}-$ радиус Шварцшильда.

Внутреннее решение Шварцшильда для однородного шара радиуса $а$ описывается интервалом

$d s^{2}=c^{2}\left(\frac{3}{2} \sqrt{1-q a^{2}}-\frac{1}{2} \sqrt{1-q W^{2}}\right)^{2} d t^{2}-\left(1-q W^{2}\right)^{-1} d W^{2}+W^{2}\left(d \theta^{2}+\sin ^{2} \theta d \phi^{2}\right)$,

здесь $q=(1 / 3) \varkappa \rho=(2 G M) /\left(c^{2} a^{3}\right), \quad \varkappa=(8 \pi G) / c^{2}, \quad \rho=(3 M) /\left(4 \pi a^{3}\right)$.

Общее свойство внешнего и внутреннего решений проявляется в том, что при определенном значении $W$ метрические коэффициенты при дифференциале $d t^{2}$ в интервалах (1) и (2) обращаются в нуль. Обращение в нуль метрического коэффициента $U$ при $d t^{2}$ означает, что гравитационное поле своим действием может не только замедлить ход времени, но даже и остановить течение времени. Для внешнего решения обращение в нуль метрического коэффициента $U$ происходит при равенстве $W=W_{g}$. Чтобы исключить такую возможность, которую теория не запрещает, мы вынуждены предположить, что радиус тела удовлетворяет неравенству

$$
a>W_{g} .
$$

*Институт физики высоких энергий, Протвино, Московская обл., Россия. E-mail: gershtein@ihep.ru; Anatoly.Logunov@ihep.ru 
Для внутреннего решения это происходит при равенстве

$$
W^{2}=9 a^{2}-8 \frac{a^{3}}{W_{g}} .
$$

Чтобы исключить возможность обращения в нуль метрического коэффициента $U$ внутри тела, мы вынуждены предположить, что

$$
a>\frac{9}{8} W_{g}
$$

Следует подчеркнуть, что неравенства (3) и (5) не являются следствием ОТО.

Внутреннее решение Шварцшильда несколько формально, но интересно прежде всего тем, что оно является точным решением уравнений ОТО. В работах [2] на примере внешнего решения Шварцшильда показано, что в релятивистской теории гравитации (РТГ) как полевой теории неравенство (3) возникает из-за гравитационной эффективной силы отталкивания, которая проявляется благодаря массе покоя гравитона из-за остановки процесса замедления хода времени. Ниже мы в рамках РТГ рассмотрим внутреннее решение типа Шварцшильда.

Внутреннее решение Шварцшильда возникло на основании уравнений ГильбертаЭйнштейна

$$
\begin{gathered}
1-\frac{d}{d W}\left[\frac{W}{V}\right]=\varkappa W^{2} \rho, \\
1-\frac{1}{V}-\frac{W}{U V} \frac{d U}{d W}=-\varkappa \frac{W^{2}}{c^{2}} p .
\end{gathered}
$$

Поскольку согласно (2) метрические коэффициенты равны

$$
U=\left(\frac{3}{2} \sqrt{1-q a^{2}}-\frac{1}{2} \sqrt{1-q W^{2}}\right)^{2}, \quad V=\left(1-q W^{2}\right)^{-1},
$$

находим

$$
\frac{{ }^{\prime}}{U}=\frac{q W}{\sqrt{1-q W^{2}}\left(3 \sqrt{1-q a^{2}} / 2-\sqrt{1-q W^{2}} / 2\right)}, \quad I^{\prime}=\frac{d U}{d W}
$$

Подставляя соотношения (7) и (8) в уравнение (6), получим выражение для давления:

$$
\frac{p}{c^{2}}=\frac{\rho}{2} \frac{\left(\sqrt{1-q W^{2}}-\sqrt{1-q a^{2}}\right)}{\sqrt{U}} .
$$

Отсюда, в частности, видно, что если бы не было исключено равенство (4), то давление внутри тела на окружности, определяемой этим равенством, обратилось бы в бесконечность. Сингулярность, которая возникает из-за обращения метрического коэффициента $U$ в нуль, нельзя устранить выбором системы координат, поскольку ее также имеет и скалярная кривизна $R$ :

$$
R=-8 \pi G \frac{3 \sqrt{1-q a^{2}}-2 \sqrt{1-q W^{2}}}{\sqrt{U}} .
$$


Покажем теперь на примере внутреннего решения типа Шварцшильда, что в РТГ благодаря силе отталкивания, которая возникает из-за остановки процесса замедления хода времени, ситуация принципиально изменяется. Тот же механизм самоограничения поля, который в РТГ [2] привел к неравенству (3) во внешнем решении Шварцшильда, приведет к неравенству типа (5) для внутреннего решения Шварцшильда.

Уравнения РТГ для метрики, определяемой интервалом

$$
d s^{2}=c^{2} U(W) d t^{2}-V(W) r^{2} d W^{2}-W^{2}\left(d \theta^{2}+\sin ^{2} \theta d \phi^{2}\right)
$$

(здесь $r=d r / d W)$, принимают вид [3]

$$
\begin{gathered}
1-\frac{d}{d W}\left[\frac{W}{V \dot{r}^{2}}\right]+\frac{1}{2}\left(\frac{m_{g} c}{\hbar}\right)^{2}\left[W^{2}-r^{2}+\frac{W^{2}}{2}\left(\frac{1}{U}-\frac{1}{V}\right)\right]=\varkappa W^{2} \rho, \\
1-\frac{1}{V \dot{r}^{2}}-\frac{W}{U V \dot{r}^{2}} U^{\prime}+\frac{1}{2}\left(\frac{m_{g} c}{\hbar}\right)^{2}\left[W^{2}-r^{2}-\frac{W^{2}}{2}\left(\frac{1}{U}-\frac{1}{V}\right)\right]=-\varkappa W^{2} \frac{p}{c^{2}} \\
\frac{d}{d W}\left[\sqrt{\frac{U}{V}} W^{2}\right]=2 r \sqrt{U V} \dot{r} .
\end{gathered}
$$

Введем новую переменную $Z=\left(U W^{2}\right) /\left(V r^{2}\right)$ и, складывая уравнения (12) и (13), получим

$$
1-\frac{1}{2 U W} Z^{\prime}+\frac{m^{2}}{2}\left(W^{2}-r^{2}\right)=\frac{1}{2} \varkappa W^{2}\left(\rho-\frac{p}{c^{2}}\right) .
$$

Вычитая уравнение (13) из уравнения (12), находим

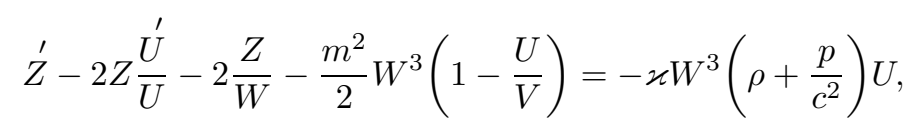

где $m=\left(m_{g} c\right) / \hbar$.

В нашей задаче компоненты тензора энергии-импульса вещества равны

$$
T_{0}^{0}=\rho, \quad T_{1}^{1}=T_{2}^{2}=T_{3}^{3}=-\frac{p(W)}{c^{2}} .
$$

Уравнение вещества

$$
\nabla_{\nu}\left(\sqrt{-g} T_{\mu}^{\nu}\right)=\partial_{\nu}\left(\sqrt{-g} T_{\mu}^{\nu}\right)+\frac{1}{2} \sqrt{-g} T_{\sigma \nu} \partial_{\mu} g^{\sigma \nu}=0
$$

для данной задачи сводится к следующему виду:

$$
\frac{1}{c^{2}} \frac{d p}{d W}=-\left(\rho+\frac{p}{c^{2}}\right) \frac{1}{2 U} \frac{d U}{d W} .
$$

Поскольку давление возрастает по направлению к центру шара, это приводит к неравенству

$$
\frac{d U}{d W}>0
$$

которое свидетельствует, что по мере приближения к центру шара функция $U$ убывает, а следовательно, идет замедление хода времени по сравнению с инерциальным. 
Поскольку во внутренней задаче Шварцшильда плотность $\rho$ принята постоянной, уравнение (16) легко решается:

$$
\rho+\frac{p}{c^{2}}=\frac{\alpha}{\sqrt{U}}
$$

Сравнивая (9) и (18), находим постоянную $\alpha$ :

$$
\alpha=\rho \sqrt{1-q a^{2}}
$$

Уравнения (14) и (15) в предположении, что

$$
m^{2}\left(W^{2}-r^{2}\right) \ll 1, \quad \frac{U}{V} \ll 1
$$

и после введения независимой переменной $y=W^{2}$ принимают вид (здесь мы обозначаем $\left.Z^{\prime}=d Z / d y\right)$

$$
\begin{gathered}
Z^{\prime}=U(1-3 q y)+\frac{\alpha \varkappa}{2} y \sqrt{U} \\
\sqrt{U} Z^{\prime}-\frac{1}{y} Z \sqrt{U}-4 Z(\sqrt{U})^{\prime}+\frac{\alpha \varkappa}{2} y U-\frac{m^{2}}{4} y \sqrt{U}=0 .
\end{gathered}
$$

В работах [2] при анализе внешнего сферически-симметричного решения Шварцшильда мы увидели, что благодаря гравитационной эффективной силе отталкивания метрический коэффициент $U$, определяющий замедление хода времени по сравнению с инерциальным, даже в сильном гравитационном поле не обращается в нуль.

Именно поэтому ниже мы будем исследовать поведение решения этих уравнений в области малых значений $y$. При массе гравитона, равной нулю, из выражения (7) для малых значений переменной $у$ имеем

$$
\sqrt{U} \sim \frac{1}{2}\left(3 \sqrt{1-q a^{2}}-1\right)+\frac{q y}{4}+\frac{1}{16} q^{2} y^{2} .
$$

Из выражения (22) также видно, что функция $\sqrt{U}$ для внутреннего решения Шварцшильда может стать равной нулю, если

$$
3 \sqrt{1-q a^{2}}=1
$$

что и приводит к бесконечному значению в центре шара как давления $p$, так и скалярной кривизны $R$. Поскольку при наличии массы покоя гравитона уравнения $(20),(21)$ останавливают процесс замедления хода времени, то естественно ожидать, что равенство (23) не может иметь места в физической (вещественной) области для функции $\sqrt{U}$. На основании $(22)$ будем искать решение уравнений $(20),(21)$ для функции $\sqrt{U}$ в форме

$$
\sqrt{U}=\beta+\frac{q y}{4}+\frac{1}{16} q^{2} y^{2}
$$

где $\beta$ - неизвестная постоянная, которую необходимо определить, используя уравнения (20), (21). 
Подставляя выражение (24) в уравнение (20) и интегрируя, находим

$$
Z=\beta^{2} y+\frac{y^{2}}{2}\left(\frac{\beta q}{2}-3 \beta^{2} q+\frac{\alpha \varkappa \beta}{2}\right)+\frac{y^{3}}{3}\left[\frac{q^{2}}{8}\left(\beta+\frac{1}{2}\right)-\frac{3 \beta}{2} q^{2}+\frac{\alpha \varkappa q}{8}\right] .
$$

Учитывая выражения (24) и (25) в уравнении (21) и пренебрегая малыми членами порядка $(m y)^{2}$, получим для определения постоянной $\beta$ уравнение

$$
2 \beta^{2} q+\beta(q-\alpha \varkappa)+\frac{m^{2}}{3}=0 .
$$

Заметим в качестве пояснения, что член, содержащий $y^{2}$, можно записать как

$$
-\frac{q y^{2}}{48}\left\{7\left[2 \beta^{2} q+\beta(q-\alpha \varkappa)\right]+3 m^{2}\right\}
$$

и, принимая во внимание уравнение (26), привести к виду

$$
-\frac{q}{72} m^{2} y^{2}
$$

Учитывая, что по определению

$$
\alpha \varkappa-q=\frac{\varkappa \rho}{3}\left(3 \sqrt{1-q a^{2}}-1\right),
$$

из уравнения (26) находим

$$
\beta=\frac{3 \sqrt{1-q a^{2}}-1+\left[\left(3 \sqrt{1-q a^{2}}-1\right)^{2}-\left(8 m^{2}\right) /(\varkappa \rho)\right]^{1 / 2}}{4} .
$$

Таким образом, метрический коэффициент $U$, определяющий процесс замедления хода времени по сравнению с инерциальным, отличен от нуля.

Если массу покоя гравитона положить равной нулю, выражение (27), как и следовало ожидать, точно совпадает с постоянным членом выражения (22). Из формулы (27) можно определить минимальное значение величины $\beta$ :

$$
\beta_{\min }=\left(\frac{m^{2}}{2 \varkappa \rho}\right)^{1 / 2}
$$

Величина $\beta$ в функции $\sqrt{U}$ определяет границу процесса замедления хода времени гравитационным полем шара. Это означает, что дальнейшее замедление хода времени гравитационным полем невозможно. Именно поэтому скалярная кривизна, определяемая выражением (10), в отличие от ОТО будет всюду конечна. Таким образом, само гравитационное поле благодаря массе покоя гравитона останавливает процесс замедления хода времени.

Согласно (27), выполнение равенства (23) благодаря наличию массы покоя гравитона невозможно, поскольку имеет место неравенство

$$
3 \sqrt{1-q a^{2}}-1 \geqslant 2 \sqrt{2}\left(\frac{m^{2}}{\varkappa \rho}\right)^{1 / 2} .
$$


Принимая во внимание верное по определению равенство

$$
q a^{2}=\frac{W_{g}}{a}
$$

на основании неравенства (29) для $\varkappa \rho \gg m^{2}$ находим

$$
a \geqslant \frac{9}{8} W_{g}\left(1+\sqrt{\frac{m^{2}}{2 \varkappa \rho}}\right) .
$$

Это ограничение на радиус тела, возникающее при изучении внутреннего решения, более сильное, чем ограничение (3), полученное в работах [2] при анализе внешнего решения. Неравенство (30), как мы видим, непосредственно следует из теории, тогда как в ОТО, чтобы избежать бесконечного давления внутри тела, неравенство (5) вынуждены вводить дополнительно. На основании (18) и (19) находим для давления выражение

$$
\frac{p}{c^{2}}=\frac{-\rho \sqrt{U}+\rho \sqrt{1-q a^{2}}}{\sqrt{U}} .
$$

Учитывая равенство (28), получим максимальное давление в центре шара:

$$
\frac{p}{c^{2}} \simeq \rho\left[\frac{2 \varkappa \rho}{m^{2}}\left(1-q a^{2}\right)\right]^{1 / 2} .
$$

Давление в центре шара конечно, тогда как в ОТО согласно (2) оно бесконечно.

Наличие в РТГ эффективной силы отталкивания, возникающей в сильных гравитационных полях, принципиально отличает ее от ОТО Эйнштейна и от ньютоновской теории гравитации, в которых господствуют только силы притяжения. В полевой теории гравитации наличие массы покоя гравитона и фундаментальное свойство гравитационного поля замедлять ход времени физического процесса по сравнению с инерциальным временем приводят к тому, что гравитационная сила может быть не только силой притяжения, но при определенных условиях (в сильных полях) и эффективной силой отталкивания. Эффективная сила отталкивания останавливает процесс замедления хода времени гравитационным полем. Гравитационное поле, таким образом, в принципе не может остановить ход времени физического процесса, поскольку оно обладает фундаментальным свойством самоограничения. Именно это свойство гравитационного поля кардинально изменяет картину эволюции материи по сравнению с ОТО, исключая возможность образования черных дыр как нефизических объектов.

Благодарности. В заключение авторы выражают благодарность В. А. Петрову и В. В. Киселеву за ценные обсуждения.

\section{Список литературы}

[1] K. Schwarzschild, Sitz. Preuss. Akad. Wiss., 1916, 189; 424.

[2] С. С. Герштейн, А.А. Логунов, М.А. Мествиришвили, Докл. РАН, 402 (2005), 34; TMФ, 2005:2 (2004), 272.

[3] А. А. Логунов, М. А. Мествиришвили, Релятивистская теория гравитации, Наука, М., 1989; А.А. Логунов, Теория гравитационного поля, Наука, М., 2001; gr-qс/0210005. 